\title{
Estudio de resistencia a la rifampicina y la dapsona en tres pacientes con recurrencia de lepra
}

\author{
Elkin Hernández, ${ }^{1}$ Nora Cardona-Castro, ${ }^{2}$ Gerzaín Rodríguez, ${ }^{1}$ \\ Sonia Villegas, ${ }^{1}$ Camilo Beltrán, ${ }^{2}$ Miyako Kimura, ${ }^{3}$ \\ Varalakshmi D. Vissa ${ }^{3}$ y Yenny Gómez ${ }^{1}$
}

Forma de citar

Hernández E, Cardona-Castro N, Rodríguez G, Villegas S, Beltrán C, Kimura M, et al. Estudio de resistencia a la rifampicina y la dapsona en tres pacientes con recurrencia de lepra. Rev Panam Salud Publica. 2008;23(2):73-7.

RESUMEN Objetivo. Detectar la presencia de cepas de Mycobacterium leprae resistentes a la rifampicina y la dapsona en tres pacientes con recurrencia de lepra y sospecha clínica de resistencia antimicrobiana, mediante la aplicación de técnicas moleculares.

Métodos. Se realizó un estudio descriptivo retrospectivo en tres pacientes multibacilares del Sanatorio de Agua de Dios, Cundinamarca, Colombia, que habian presentado recidivas de lepra documentadas por su historia clínica, baciloscopia y biopsia. Se obtuvieron biopsias de lesiones cutáneas que se procesaron para la extracción y purificación del ADN bacilar. Se amplificaron regiones de los genes $\mathrm{rpoB} y$ folP1 asociadas con la resistencia antimicrobiana, mediante la reacción en cadena de la polimerasa "touch-down" y se secuenciaron los productos amplificados mediante el método de Sanger.

Resultados. Se detectó una mutación puntual en el nucleótido 1367 del gen rpoB en dos de las muestras estudiadas. No se encontró la mutación estudiada en el gen folP1 en ninguno de los tres pacientes.

Conclusiones. La mutación identificada demostró la presencia de bacilos de M. leprae resistentes a la rifampicina en dos de los tres pacientes estudiados con recurrencia de la enfermedad. No se detectó la mutación indicadora de resistencia a la dapsona en ninguno de los tres pacientes.

Palabras clave Mycobacterium leprae, lepra, resistencia microbiana a las drogas, rifampicina, dapsona, reacción en cadena de la polimerasa, Colombia.

1 Universidad de La Sabana, Facultad de Medicina, Chía, Colombia. La correspondencia se debe dirigir a Yenny Milena Gómez, Universidad de La Sabana, Campus Universitario Puente del Común, Autopista Norte km 21, Chía, Colombia. Correo electrónico: yenny.gomez1@unisabana.edu.co

2 Instituto Colombiano de Medicina Tropical-CES, Sabaneta, Colombia.

3 Colorado State University, Department of Microbiology, Immunology, and Pathology, Fort Collins, Colorado, Estados Unidos de América.
Hasta 1982, el tratamiento de la lepra se basó en la monoterapia con dapsona. La emergencia de cepas de Mycobacterium leprae resistentes a este antimicrobiano (1) hizo que la Organización Mundial de la Salud (OMS) recomendara el uso de la poliquimioterapia (PQT), que combina tres anti- bióticos: dapsona, clofazimina y rifampicina (2). La base de la PQT es la rifampicina, ya que es el componente con mayor actividad bactericida, incluso mayor que la de nuevos antimicrobianos empleados específicamente contra $M$. leprae $(3,4)$. La función principal de la dapsona y la clofazimina es 
garantizar la eliminación de las poblaciones de $M$. leprae resistentes a la rifampicina (3). El uso de este esquema terapéutico ha permitido reducir eficazmente la prevalencia global de la enfermedad, aunque en los países con mayor endemia, la incidencia ha permanecido estable (5) y se ha observado una mayor frecuencia de la recurrencia de la enfermedad. Esta recurrencia se ha constatado en pacientes con lepra multibacilar que presentaban un alto índice de infección al inicio del tratamiento y que se siguieron durante varios años después de terminada la PQT $(6,7)$.

También se ha documentado la presencia de cepas de $M$. leprae resistentes a los antimicrobianos empleados en la PQT, entre ellos la rifampicina (8-11). Aunque esta modalidad terapéutica ha limitado la diseminación de la resistencia a la dapsona, se siguen notificando casos resistentes a este medicamento, incluso en áreas donde la PQT se ha aplicado exitosamente $(8,12,13)$. Además, se ha informado de posibles cepas de $M$. leprae resistentes a múltiples antimicrobianos, responsables de la recurrencia en casos tratados con monoterapia y PQT $(9,11)$.

La OMS ha planteado la necesidad de vigilar la emergencia de la resistencia a los antimicrobianos, principalmente a la rifampicina, ya que esto puede reducir significativamente la eficacia del tratamiento y obstaculizar la erradicación de la lepra $(10,14)$.

La prueba estándar para determinar la susceptibilidad antimicrobiana de M. leprae es la técnica de la almohadilla plantar del ratón, que además de costosa, demora entre 6 y 12 meses en dar resultados y está disponible en pocos laboratorios (1). Debido a estas limitaciones, se han desarrollado diferentes técnicas moleculares basadas en la detección de mutaciones puntuales en los genes rpoB y folP1 de $M$. leprae, las cuales se utilizan como marcadores de resistencia a la rifampicina y la dapsona, con una alta correlación con la prueba estándar $(8-11,15,16)$. Esto se basa en diversas investigaciones que han demostrado que esas mutaciones puntuales en los genes rpoB y folP1 son las responsables de la resistencia de $M$. leprae a la rifampicina y la dapsona, respectivamente $(8,15,16)$. Se ha podido predecir la resistencia antimicrobiana de algunos aislamientos mediante diversa técnicas de biología molecular que detectan estas mutaciones (9-11).

En el municipio de Agua de Dios, departamento de Cundinamarca, Colombia, donde funcionó un sanatorio de reclusión obligatoria para enfermos de lepra entre 1872 y 1961, se han presentado casos de recurrencia de la enfermedad en los que han concurrido factores de riesgo para la aparición de cepas resistentes de M. leprae (17). El objetivo de este estudio fue detectar la presencia de cepas de $M$. leprae resistentes a la rifampicina y la dapsona en tres pacientes de ese municipio con recurrencia de lepra y sospecha clínica de resistencia antimicrobiana, mediante la aplicación de técnicas moleculares.

\section{MATERIALES Y MÉTODOS}

\section{Pacientes}

Se realizó un estudio descriptivo retrospectivo en tres pacientes del Sanatorio de Agua de Dios, de Cundinamarca, Colombia, que presentaron de uno a tres episodios de recurrencia de la enfermedad, por lo que se sospechaba que estaban infectados por una cepa resistente a antibióticos (17). Todos los pacientes habían recibido monoterapia con dapsona por más de dos décadas, pero de forma irregular, con dosis variables y no supervisadas, y los pacientes 1 y 2 habían recibido además PQT administrada en iguales condiciones. En el cuadro 1 se muestran los datos principales de los tres pacientes.

\section{Obtención y procesamiento de las muestras}

De cada paciente con recurrencia -documentada por su historia clínica, baciloscopia y biopsia- se tomó una muestra de las lesiones cutáneas que se conservó en etanol al 70\% (18). Esta muestra se utilizó para el análisis molecular de la cepa infectante mediante la reacción en cadena de la polimerasa (RCP) en el Laboratorio del Grupo de Microbiología Molecular de la Universidad de La Sabana, Chía, Colombia. La extracción y la purificación del ADN bacilar se realizaron por los métodos de choque térmico (19) y digestión enzimática con proteinasa $\mathrm{K}$, respectivamente, con el estuche DNeasy ${ }^{\circledR}$ Tissue (Qiagen $\mathrm{GmbH}$, Hilden, Alemania). Se estandarizó la técnica de RCP denominada touch-down para amplificar regiones de los genes rpoB y folP1 asociadas con la resistencia a la rifampicina y la dapsona, respectivamente (8-11, 15, 16). Para el gen $r p o B$ se utilizaron los cebadores descritos previamente (15), que amplifican un fragmento de 394 pares de bases $(\mathrm{pb})$, y para el gen

CUADRO 1. Características clínicas de los pacientes con recurrencia de lepra estudiados, Colombia

\begin{tabular}{|c|c|c|c|c|c|c|}
\hline Caso & Edad & Sexo & $\begin{array}{l}\text { Diagnóstico } \\
\text { inicial, año }\end{array}$ & $\begin{array}{l}\text { Tipo y período de } \\
\text { tratamiento previo }\end{array}$ & $\begin{array}{c}\text { No. de recurrencias } \\
\text { y años de } \\
\text { su diagnóstico }\end{array}$ & $\begin{array}{l}\text { Diagnóstico de } \\
\text { la recurrenciab }\end{array}$ \\
\hline 1 & 57 & $M^{c}$ & $\mathrm{LL}^{d}, 1958$ & $\begin{array}{l}\text { DDS }^{1960-1982} \\
\text { PQT }^{f} 1983-1997\end{array}$ & $3(1999,2002,2005)$ & LL \\
\hline 2 & 69 & M & LL,1954 & $\begin{array}{l}\text { DDS 1954-1982 } \\
\text { PQT 1982-1984 }\end{array}$ & $1(2005)$ & LL \\
\hline 3 & 62 & M & LL, 1961 & $\begin{array}{l}\text { DDS 1961-1984 } \\
\text { PQT: desconocido }\end{array}$ & $1(2004)$ & LL \\
\hline
\end{tabular}

\footnotetext{
a Tratamiento irregular, con dosis variables y no supervisado.

b Diagnóstico por historia clínica, baciloscopia y biopsia.

c Masculino.

d Lepra lepromatosa.

e Dapsona.

f Poliquimioterapia.
} 
folP1 se utilizaron los iniciadores 5'CAGGACGTCGAGGCGATCAC-3' y 5'-TCCTCGTCAGCGGTCAAGTA-3', que amplifican un fragmento de 389 pb. Se emplearon las mismas concentraciones de reactivos y condiciones del termociclador para ambas reacciones.

La RCP se realizó en un volumen final de $25 \mu \mathrm{L}$ de solución tampón de $\mathrm{MgCl}_{2}$ 1,5 mM que contenía 1 pM de cada iniciador, $2 \mu \mathrm{M}$ de cada trifosfato de desoxinucleósido, 2,5 unidades (U) de polimerasa Taq (Promega, Madison, Wisconsin, Estados Unidos de América) y $5 \mu \mathrm{L}$ de muestra. Se empleó un termociclador iCycler (BioRad, Hercules, California, Estados Unidos) con el siguiente programa: 5 min de desnaturalización inicial a $95^{\circ} \mathrm{C}$; 11 ciclos de 1 min de desnaturalización a $95^{\circ} \mathrm{C}, 1 \mathrm{~min}$ de anillamiento a $68^{\circ} \mathrm{C}$ (con una disminución de $1{ }^{\circ} \mathrm{C}$ por cada ciclo hasta llegar a $58{ }^{\circ} \mathrm{C}$ ) y 2 min de extensión a $72{ }^{\circ} \mathrm{C}$; seguidos de 27 ciclos de 1 min de desnaturalización a $95^{\circ} \mathrm{C}, 1 \mathrm{~min}$ de anillamiento a $57{ }^{\circ} \mathrm{C}$ y 2 min de extensión a $72{ }^{\circ} \mathrm{C}$; $\mathrm{y}$ un paso final de extensión por 30 segundos a $72{ }^{\circ} \mathrm{C}$. Como control positivo se empleó el ADN bacilar extraído de una biopsia de piel de un paciente con diagnóstico confirmado de lepra lepromatosa por historia clínica, baciloscopia y biopsia. Como control negativo de la reacción se usó agua ultrapura y ADN extraído de una biopsia de piel de un paciente con diagnóstico de cicatriz hipertrófica, sin signos ni antecedentes de lepra. Los productos de la RCP se verificaron por electroforesis en gel de agarosa al $2 \%$ coloreado con $0,2 \mu \mathrm{g} / \mathrm{mL}$ de bromuro de etidio y se visualizaron en un sistema fotodocumentador con luz ultravioleta (GelDoc XR, Bio-Rad).

\section{Secuenciación del ADN}

Se secuenció el producto de la amplificación mediante el método de Sanger (20) con un secuenciador automático ABI Prism (Applied Biosystems, Foster City, California, Estados Unidos). Los cromatogramas se editaron con el programa Chromas v. 1.45 (Technelysium, Queensland, Australia) y el análisis bioinformático se realizó mediante el alineamiento local con el módulo Water del programa Emboss (21), usando como patrón de comparación las secuencias de una cepa sensible a la dapsona y de otra sensible a la rifampicina (códigos de acceso Z14314 y AB028656, respectivamente, en la base de datos GenBank). Se identificó la posición de los nucleótidos sustituidos mediante los tres sistemas de referencia descritos $(15,22$, 23) y se compararon con las mutaciones publicadas $(8-11,15,16)$.

\section{RESULTADOS}

\section{Genotipificación de los genes rpoB y folP1 de M. leprae}

En las muestras de los pacientes 1 y 2 se detectó una mutación puntual en el gen $r p o B$, localizada en el nucleótido 1367 (TCG $\rightarrow$ TTG), que produce un cambio de serina por leucina en la posición 456 de la secuencia aminoacídica, según el sistema de numeración empleado en la base de datos Leproma (23) (cuadro 2). No se observaron cambios en la secuencia del gen rpoB de $M$. leprae en el paciente 3 , ni en la secuencia del gen folP1 en ninguna de las tres muestras estudiadas. El control positivo empleado mostró ADN de M. leprae sin mutaciones en las regiones estudiadas de los genes rpoB y folP1. Los controles negativos no evidenciaron la presencia del ADN bacilar.

\section{DISCUSIÓN}

En este estudio se demostró por primera vez la presencia de cepas de $M$. leprae resistentes a la rifampicina en Colombia. Además. A pesar de haberse documentado en las historias clínicas de los tres pacientes estudiados el tratamiento inadecuado con dapsona, no se encontró la mutación estudiada de resistencia a este medicamento.

La técnica empleada, basada en la RCP con secuenciación del ADN bacilar amplificado (24), es la más confiable para identificar mutaciones y en esta investigación permitió detectar una mutación en el gen $r p o B$ en las cepas de $M$. leprae que portaban los pacientes 1 y 2 . Esta modificación, descrita inicialmente por Honoré y Cole (15), es específica de las cepas que poseen resistencia a la rifampicina $y$, como lo han confirmado otros autores mediante la técnica de la almohadilla plantar del ratón, esta es la mutación que se presenta con mayor frecuencia (24). Los resultados de los estudios moleculares concuerdan con los ante-

CUADRO 2. Mutación detectada (elementos subrayados) en el gen rpoB de Mycobacterium leprae en las muestras de los pacientes 1 y 2, Colombia

\begin{tabular}{|c|c|c|c|c|c|}
\hline \multirow[b]{2}{*}{ Tipo de secuencia } & \multicolumn{2}{|c|}{$\begin{array}{c}\text { Secuencia del gen rpoB de } M \text {. leprae } \\
\text { y sus aminoácidos deducidos }\end{array}$} & \multicolumn{3}{|c|}{$\begin{array}{l}\text { Posición de la mutación, según } \\
\text { diversos sistemas de numeración }\end{array}$} \\
\hline & $\begin{array}{l}\text { Cepas sensibles a } \\
\text { la rifampicina }\end{array}$ & Muestras estudiadas & $\begin{array}{l}\text { Telenti } \\
\text { y col. }\end{array}$ & $\begin{array}{l}\text { Honoré } \\
\text { y Cole }\end{array}$ & Leproma $^{\mathrm{C}}$ \\
\hline Nucleotídica & CGG CTG TㅌG GCG CTG & CGG CTG TIG GCG CTG & 1592 & 1274 & 1367 \\
\hline Aminoacídica & Arg Leu Ser Ala Leu & Arg Leu Leu Ala Leu & 531 & 425 & 456 \\
\hline
\end{tabular}


cedentes y la evolución clínica de los pacientes: un tratamiento irregular y la recurrencia de la enfermedad, condiciones asociadas con el desarrollo de la resistencia a antimicrobianos y con la resistencia secundaria a la rifampicina $(2,9,11,24)$. El paciente 3 no había recibido rifampicina, lo que es consecuente con el hecho de que en las muestras de su biopsia no se detectó la mutación en el gen rpoB, propia de cepas con resistencia a ese antibiótico.

La detección de mutaciones puntuales en el gen folP1 ha sido identificada con una alta frecuencia en las cepas de $M$. leprae que presentan resistencia alta o intermedia a la dapsona $(8,12,25,26)$. A pesar de que estos tres pacientes recibieron tratamiento con dapsona de manera prolongada e irregular, en sus muestras no se detectaron mutaciones en la región secuenciada del gen folP1, lo cual podría indicar que aún son sensibles a la dapsona. No obstante, la evolución clínica de estos pacientes indica que presentan resistencia secundaria a la dapsona. Varias hipótesis podrían explicar este resultado: la presencia de bacilos con una baja resistencia, no detectable mediante las técnicas moleculares, que no se correlaciona con las manifestaciones clínicas $(8,13,25,26)$; la presencia de porinas y mecanismos de eflujo que permiten el transporte del medicamento al exterior de la bacteria, como se ha descrito en otras micobacterias $(27,28)$; o que las muestras tuvieran una mezcla de bacilos sensibles y resistentes en diferentes proporciones y que la amplificación y secuenciación del genotipo dominante correspondiera al de los bacilos sensibles a la dapsona, como han sugerido otros autores $(16,25)$.

A pesar del reducido número de casos estudiados, los presentes resultados permiten afirmar que la metodología molecular empleada puede ayudar a detectar tempranamente una posible recidiva. Esta metodología permitió estudiar sendas regiones de los genes rpoB y folP1 que contienen mutaciones asociadas con la resistencia antimicro- biana. Sin embargo, existen otras regiones génicas que podrían presentar mutaciones relacionadas con la resistencia a antibióticos y que deben ser objeto de estudio.

Debido a la frecuencia con que se ha observado la recidiva de lepra en pacientes tratados con monoterapia de dapsona durante años (17) es recomendable realizar un seguimiento clínico y baciloscópico anual a fin de detectar tempranamente las posibles recidivas.

Agradecimientos. El presente estudio recibió financiamiento del Fondo Patrimonial Especial de La Universidad de La Sabana, Chía, Colombia, y del Instituto Colombiano de Medicina Tropical del Instituto de Ciencias de la Salud (ICMT-CES) y el convenio CSUICMT-NIH/NIAID RO1-AI5-37720. Los autores agradecen al personal de salud del Sanatorio de Agua de Dios y a Tiberio Rodríguez, auxiliar técnico del Programa de Lepra, por su apoyo en la búsqueda de las historias clínicas.

\section{REFERENCIAS}

1. Ji B. Rifampicin resistant leprosy: a review and a research proposal of a pilot study. Lepr Rev. 2002;73:2-8.

2. World Health Organization Study Group. Chemotherapy of leprosy for control programmes. Geneva: WHO; 1982. (WHO Technical Report Series No. 675).

3. Ji B, Perani EG, Petinom C, Grosset JH. Bactericidal activities of combinations of new drugs against Mycobacterium leprae in nude mice. Antimicrob Agents Chemother. 1996;40: 393-9.

4. Ji B, Sow S, Perani E. Bactericidal activities of a single-dose combination of ofloxacin plus minocycline, with or without rifampin, against Mycobacterium leprae in mice and in lepromatous patients. Antimicrob Agents Chemother. 1998;42:1115-20.

5. Lockwood D, Suneetha S. Leprosy: too complex a disease for a simple elimination paradigm. Bull World Health Organ. 2005;83: 230-5.

6. Gelber RH, Balagon MVF, Cellona RV. The relapse rate in MB leprosy patients treated with 2 years of WHO-MDT is not low. Int J Lepr Other Mycobact Dis. 2004;72:493-500.

7. Cellona RV, Balagon MVF, dela Cruz EC, Burgos JA, Abalos RM, Walsh GP, et al. Longterm efficacy of 2-year WHO multiple-drug therapy (MDT) in multibacillary (MB) leprosy patients. Int J Lepr Other Mycobact Dis. 2003; 71:308-19.

8. Kai M, Matsuoka M, Nakata N, Maeda S, Gidoh M, Maeda Y, et al. Diaminodiphenylsulfone resistance of Mycobacterium leprae due to mutations in the dihydropteroate synthase gene. FEMS Microbiol Lett. 1999;177: 231-5.

9. You E, Kang T, Kim S, Lee S, Chae G. Mutations in genes related to drug resistance in Mycobacterium leprae isolates from leprosy patients in Korea. J Infect. 2005;50:6-11.

10. Cambau E, Bonnafous P, Perani E, Sougakoff W, Ji B, Jarlier V. Molecular detection of rifampin and ofloxacin resistance for patients who experience relapse of multibacillary leprosy. Clin Infect Dis. 2002;34:39-45.

11. Maeda S, Matsuoka M, Nakata N, Kai M, Maeda Y, Hashimoto K, et al. Multidrug resistant Mycobacterium leprae from patients with leprosy. Antimicrob Agents Chemother. 2001; 45:3635-9.

12. Sekar B, Elangeswaran N, Jayarama E, Rajendran M, Kumar S, Vijayaraghavan R, et al. Drug susceptibility of Mycobacterium leprae: a retrospective analysis of mouse foodpad inoculation results from 1993 to 1997. Lepr Rev. 2002;73:239-44.

13. Dela Cruz E, Cellona R, Balagon M, Villahermosa LG, Fajardo TT, Hablaos RM, et al.
Primary dapsone resistance in Cebu, the Philippines: cause for concern. Int J Lepr Other Mycobact Dis. 1996;64:264-7.

14. World Health Organization. Global strategy for further reducing the leprosy burden and sustaining leprosy control activities (plan period: 2006-2010). Geneva: WHO; 2005. Hallado en: http://www.who.int/lep/ resources/GlobalStrategy.pdf. Acceso el 17 de enero de 2008.

15. Honoré N, Cole ST. Molecular basis of rifampin resistance in Mycobacterium leprae. Antimicrob Agents Chemother. 1993;37:414-8.

16. Williams DL, Spring L, Harris E, Roche P, Gillis TP. Dihydropteroate synthase of Mycobacterium leprae and dapsone resistance. Antimicrob Agents Chemother. 2000;44:1530-7.

17. Rodríguez G, Pinto R, Laverde C, Sarmiento M, Riveros A, Valderrama J, et al. Recidivas postratamiento de la lepra multibacilar. Biomédica. 2004;24:133-9.

18. Torres P, Camarena JJ, Gómez JR, Nogueira JM, Gimeno V, Navarro JC, et al. Comparison of PCR mediated amplification of DNA and the classical methods for detection of $\mathrm{Myco-}$ bacterium leprae in different types of clinical samples in leprosy patients and contacts. Lepr Rev. 2003;74:18-30.

19. Zhang ZQ, Ishaque M. Evaluation of methods for isolation of DNA from slowly and rapidly 
growing mycobacteria. Int J Lepr Other Mycobact Dis. 1997;65:469-76.

20. Sanger F, Nicklen S, Coulson AR. DNA sequencing with chain-terminating inhibitors. Proc Natl Acad Sci. 1977;74:5463-7.

21. Rice P, Longden I, Bleasby A. EMBOSS: the European molecular biology open software suite. Trends Genet. 2000;16:276-7.

22. Telenti A, Imboden $P$, Marchesi F, Lowrie D, Cole S, Colston MJ, et al. Detection of rifampicin-resistance mutations in Mycobacterium tuberculosis. Lancet. 1993;341:647-50.

23. Jones L, Moszer I, Cole ST. Leproma: a Mycobacterium leprae genome browser. Lepr Rev. 2001;72:470-7.
24. Williams D, Gillis T. Molecular detection of drug resistance in Mycobacterium leprae. Lepr Rev. 2004;75:118-30.

25. Cambau E, Carthagena L, Chauffour A, Ji B, Jarlier V. Dihydropteroate synthase mutations in the folP1 gene predict dapsone resistance in relapsed cases of leprosy. Clin Infect Dis. 2006;42:238-41.

26. Guelpa-Lauras CC, Cartel JL, ConstantDesportes M, Millan J, Bobin P, Guidi C, et al. Primary and secondary dapsone resistance of M. leprae in Martinique, Guadeloupe, New Caledonia, Tahiti, Senegal, and Paris between 1980 and 1985. Int J Lepr Other Mycobact Dis. 1987;55:672-9.
27. Niederweiss M. Mycobacterial porins-new channel proteins in unique outer membranes. Mol Microbiol. 2003;49:1167-77.

28. Li XZ, Zhang L, Nikaido H. Efflux pumpmediated intrinsic drug resistance in $\mathrm{Myco-}$ bacterium smegmatis. Antimicrob Agents Chemother. 2004;48:2415-23.

Manuscrito recibido el 29 de enero de 2007. Aceptado para publicación, tras revisión, el 23 de noviembre de 2007.

ABSTRACT Objective. To detect the presence of rifampin- and dapsone-resistant strains of $\mathrm{Myco}^{-}$ bacterium leprae in three patients with recurring leprosy and clinically-suspected antimicrobial resistance through molecular techniques.

\section{Study of rifampin and dapsone resistance in three patients with recurring leprosy}

Key words
Methods. A retrospective, descriptive study was conducted of three multibacillary patients at the "Agua de Dios" Sanitarium in Cundinamarca, Colombia, that presented leprosy relapses that were documented by medical history, bacilloscopy, and biopsy. Biopsies were taken of the skin lesions and the bacteria were subject to DNA extraction and purification. Regions of the $r p o B$ and folP1 genes associated with antimicrobial resistance were amplified and subjected to touch-down polymerase chain reaction and the amplified products were sequenced using the Sanger method.

Results. A punctual mutation was identified in nucleotide 1367 of the rpoB gene in two of the samples studied. This mutation was not found in the folP1 gene of any of the three patients.

Conclusions. The mutation identified showed strains of rifampin-resistant M. leprae in two of the three patients with recurring leprosy. Mutations that indicate dapsoneresistance were not detected in any of the three patients.

Mycobacterium leprae, leprosy, drug resistance, rifampin, dapsone, polymerase chain reaction, Colombia. 